\title{
The Autoimmune Brain: A Five-Step Plan for Treating Chronic Pain, Depression, Anxiety, Fatigue, and Attention Disorders
}

\author{
David S Younger MD, MPH, MS
}

Department of Clinical Medicine, CUNY School of Medicine at City College, New York, NY and the Graduate School of Public Health and Health Policy, City University of New York, New, USA.

Address for Correspondence: David S Younger MD, MPH, MS; Email: youngd01@nyu.edu

Received 28 December 2019;

Accepted 30 December 2019;

Published 01 January 2020

\begin{abstract}
This year, 2019 saw the publication of The Autoimmune Brain ${ }^{1}$ that explains the background and application of I-Cubed, shorthand for post-infectious immunity and autoimmunity that is closely associated with the causes of common and rare neuropsychiatric illnesses.
\end{abstract}

Keywords: Neuropathy, Dysautonomia, Encephalopathy, Neuropsychiatric Disorders.

\section{Book Review}

An emerging concept referred to as I-Cubed ${ }^{[1]}$ describes the relationship of individuals to infectious exposures that leads to autoimmune diseases. The multiplier effect of infection, immunity and inflammation symbolized as $\mathrm{I}^{3}$, is the basis for post-infectious autoimmunity. In keeping with the dynamic nature of I-Cubed, post-infectious autoimmune illness arises when protective immunity becomes the source of autoimmunity, conditioned by environmental and genetic factors.

There are new scientific terms to describe the factors associated with I-Cubed ${ }^{[2]}$. The exposome relates all microbes in the environment with which we come in contact. The human microbiome is the sum of the microbiota, with the infectome, comprising pathogenic microbes alone. Every infectious event evokes an inflammatory immune response directed against the intruding microbe, however the response is generally selflimited and associated with full recovery. It classically involves three highly specific responses. First, the secretion of specific antibodies into the blood stream called immunoglobulins (Ig) named IgM and IgG, for the chemical composition and response to recent or longstanding infection respectively. Second, a cell-mediated inflammatory immune response comprised of activated naïve T-(thymic) and B- (bone marrow) lymphocytes. Third, the secretion of cytokines that comprise receptor specific inflammatory intermediates such as tumor necrosis factor (TNF) and interleukins (ILs) which modulate the balance between humoral and cell-mediated immunity through an intricate process of receptor signalling. Together these three responses assure removal of the infectious intruder and future immunity. Vaccination, like post-infectious immunity, guards against microbial infections that might otherwise become epidemic if the majority of the population failed to be protected or harmful to pregnant women.

In recent years, microbes have been recognized for their importance in driving the inflammatory immune response, turning it on and off at will, thus creating post-infectious illness especially in those with imperfect defences and a genetic susceptibility. A remarkable feature of I-Cube is that it can be the cause of autoimmune illness, and neuropathic pain, chronic fatigue, chronic infection, and neuropsychiatric illness that responds to immune modulation employing IVIg or subcutaneous (SC) - Ig therapy.

${ }^{1}$ https://rowman.com/ISBN/9781538117705/The-Autoimmune-Brain-A-Five-Step-Plan-for-Treating-Chronic-Pain-Depression- 
Meet Lucy ${ }^{[1]}$ (and hear her story online), ${ }^{2}$ a 34-year-old stock analyst who suffered from recurrent postpartum depression that was in remission until she awoke one evening several months after her second childbirth with pain. She remembers feeling a pain in her arms and joking to herself, "this must be what neuropathy feels like." Little did she know that over the next two months it would be the most excruciating situation she would ever endure? It felt like more than a gym injury when it attacked all of her joints with an intensity that electrified her body and made her feel continuously in a fight-or-flight mind frame. She saw a multitude of doctors in neurology, rheumatology, infectious disease, pain management, physical therapy and alternative medicine, but the best they could do was prescribe oxycodone and morphine. She was told of a positive Lyme test in the blood, but was not offered a therapeutic course of antibiotic therapy. Was it in her head she remembers thinking? When the pain worsened, she comforted herself by running cold water over her hands, eventually taking pots of cold water around the house wherever she was. She was hospitalized one last time for evaluation but still did not get a path forward, and was instead placed on an intravenous opioid drip for pain. Her husband called me asking for a meeting; and after speaking with her medical team, she came directly to my office where I performed several tests: positron emission tomography fused to magnetic resonance imaging (PET/MRI) of the brain to look for deficits in brain metabolism to explain depression; electrical studies and biopsy studies of the nerves to identify the cause of her neuropathic pain; and a tilt table test searching for an autonomic disturbance to explain her arousal state of fightor-flight. These studies showed what I postulated, a postinfectious immune and inflammatory insult affecting the nervous system. I treated her with doxycycline and weekly intravenous Ig (IVIg) infusions that led to a full recovery in several months ${ }^{[1]}$.

Infection and immunity can be difficult to diagnose when symptoms and signs are subtle or misunderstood and diagnostic studies show normal or absent findings due to a variety of reasons. Health providers may fail to identify a suspected bacteria, viral, fungal or parasitic organism especially when serological studies in the blood come back inconclusive or negative. This is often due to microbes that hide from the host immune system because of a genetic mutation, or those that hide behind cell walls or in cysts like Borrelia (B.) burgdorferi, the causative agent of Lyme disease. Herpesvirus which are the cause of shingles, mononucleosis and oral and genital herpes, have an infinity for neurons where they can dormant for years between clinical relapses. Some individuals have genetic abnormalities that disturbed normal immune activation or lack normal responsiveness due to use of immunosuppressant medication like prednisone which attenuates immune responsiveness to antigenic challenges

\footnotetext{
${ }^{2}$ http://davidsyounger.com/case-studies
}

and the false impression of a non-infective uninflamed state.

I-Cubed starts at sites of injury and inflammation which is the entry way for all antigenic threats. Patrolling cells called macrophages unique to each organ such as the brain, nerve, and lung tissues, release pro-inflammatory cytokines, typically TNF and ILs that cause vasodilation, increased capillary permeability (humoral response) and leukocyte entry into injured and infected tissues (cellular response). This results in the hallmark of pain, heat, redness and swelling of inflammation. As the inflammatory response progresses and evolves, there can be prompt resolution in a highly regulated process. However, persistence of the initiating stimulus leads to chronic inflammation with the typical dysregulation between destructive inflammatory and excessive healing responses seen in systemic autoinflammatory autoimmune diseases such as arthritis, atherosclerosis, and asthma. Corticosteroids and other immunosuppressants inhibit many of the initial events in an inflammatory response.

The mechanisms by which pathogenic microbes activate the immune system takes place in the tri-molecular complex. Foreign intruders to any tissue including the brain are tagged by antigen presenting cells (APCs) specific for that organ, and escort it to naïve T-cells and adjoining protein molecules encoded by human leukocyte antigen (HLA) genes located on chromosome $6(\mathrm{p} 21)$ in a region known as the major histocompatibility complex (MHC) class II. The interaction of these four molecules--antigen and antigen presenting cell (APC), autoreactive T-cell, and MHC class II, leads to a burst of $\mathrm{T}$-cell and B-cells activation and differentiation with secretion of cytokines and other inflammatory intermediates that comprise the systemic autoimmune response. Through the process called molecular mimicry, the body's immune cells profile and isolate antigenic molecules present on all cell surfaces that resemble the original antigen intruder, and unleash their immune attack. An excellent example is Guillain-Barre syndrome (GBS) triggered by Campylobacter jejuni infection that causes infectious diarrhea weeks before the onset of characteristic paralysis. Ganglioside antibodies cross reactive to both nerve fibers and specific targets of the microbe leads to paralytic illness due to an assault on the peripheral nerves by autoimmune inflammatory mechanism related to the preceding bacterial infection. Molecular mimicry occurs when the similarities between foreign microbial and self-peptides result in the cross-activation of autoreactive $\mathrm{T}$ - or B-lymphocytes by pathogen-derived antigens. The disease is readily reversed by instituting plasma exchange and IVIg therapy to diminish the inflammatory immune response that perpetuates the illness long after the infection has cleared.

The inadequacies of traditional techniques to isolate microbial causes of infection led to high throughput sequencing (HTS) that focuses on bits of genetic material derived from ribonucleic acid (RNA) called ribosomal 
ribonucleic acids which encode the genetic blueprint of cells and translates it into protein building blocks. Small bits of ribosomal RNA called 16S rRNA tag certain genetic molecular sequences in invading organisms with a precision far beyond traditional gram stains, cultures, and antibody levels, providing a molecular fingerprint. In a matter of minutes, HTS matches bits of microbial 16S rRNA present in blood, cerebrospinal fluid, and other tissue specimens to libraries of known microbes to identify rare infectious and epidemic neurological illnesses. Undiagnosed cases of sterile meningitis and rare encephalomyelitis, as well as, epidemics due to Ebola, Zika, influenza, and the Middle East Respiratory Syndrome have all benefited from HTS.

HTS also proved invaluable in a study of several hundred patients who underwent prosthetic joint replacement surgery and suspected of insidious prosthetic joint infection (PJI) due to chronic postoperative pain. With $16 \mathrm{~S}$ rRNA gene PCR analysis of blood the investigators were able to reclassify cases thought to be uninfected but and later found to harbor microbial genetic footprints and others placed on antibiotics for presumed infection but not so, with a precision that outperformed traditional cultivation techniques. With only two-thirds of unexplained critical illness and deaths in previously healthy persons due to infectious causes ever solved according to The Centers for Disease Control and Prevention (CDC), there will be a pressing need to make molecular genetic approaches to the detection of present and emerging infectious diseases widely available.

The concept of autoimmunity is fundamentally related to self-tolerance, defined as the ability of the immune system to prevent itself from targeting self-molecules, cells or tissues. Viral infections that are a natural part of our existence have provided insight into the development of autoimmunity through their interaction with the host immune system ${ }^{[3]}$. The breakthrough in our understanding of the role of viruses in triggering autoimmunity came from mice infected with the lymphocytic choriomeningitis virus (LCMV) that were genetically engineered to express certain protein to track the antiviral autoimmune response to infection. A single infection with LCMV led to the breakdown of tolerance to pancreatic islet cells that produces insulin and a cytotoxic T-cell-mediated form of autoimmune diabetes.

Over time, pathogenic microbial insults, referred to as the autoinfectome can be the cause of inflammation and autoimmunity ${ }^{[4]}$. One example is the repeated disruption of the intestinal milieu and the impairment of gut homeostasis as a main contributor to inflammatory bowel disease ${ }^{[5]}$. Another example is increasing evidence that finds a microbial link to juvenile arthritis that begins in early childhood when early life events influence disease risk such as infant feeding practice and antibiotic exposures that impact microbes and disease risk. Individuals who develop more than one autoimmune disease illustrate the phenomenon of the mosaicism of autoimmunity ${ }^{[6]}$ that relates the varying and unique clinical presentations due to the different combinations of factors involved in autoimmunity. Such patients present to doctors of varying specialties as a medical mystery due to their overwhelming complexity.

Several large scale epidemiologic studies focusing on the relation between autoimmune disease and severe neuropsychiatric illnesses find a role for post-infections autoimmunity through I-Cubed with the production of pathogenic autoantibodies that have targets in brain areas such as the hippocampus, where psychiatric illness develops [7]. Brain areas such as the hippocampus are highly dependent upon serotonergic neurotransmission and responsive to selective serotonin reuptake inhibitors (SSRIs) and neuroimmune modulation with Ig therapy to favor neural plasticity and neurogenesis over neuroinflammation, to restore neural circuits ${ }^{[8]}$. The recent finding of hippocampal hypometabolism in brain PET MRI of a patient with persistent symptoms of Lyme disease suggests that post-infectious immunity or I-Cubed may be the cause of neuropsychiatric complaints associated with post-treatment Lyme disease syndrome (PTLDS) ${ }^{[9]}$. Treatment with immune modulatory (Ig) therapy appears to be an effective remedy when chronic antibiotics show no further benefit.

The continued search for unrecognized pathogens of the microbiome reveals surprises about the distribution and diversity of the human genome. Since first recognition of the highly virulent human immunodeficiency virus type 1 (HIV1) more than two decades ago, genetic studies showed that all humans possesses retrovirus sequences as an integral part of their 9 genome. This likely occurred in the course of evolution when the HIV-1 ribonucleic acid genetic sequences were inserted into human germ-line reproductive cells, replicating along with other host cellular genes as part of the microbiome ${ }^{[10]}$. However, pathogenicity, which requires the budding of retroviral particles as the first step in the pathway to a new replication cycle encoded on the env gene is conspicuously absent from human microbiome genetics.

Molecular mimicry, due to shared immunologic identities or epitopes between the microbe and host, results in activation of autoreactive $\mathrm{T}$ and B-cells by pathogen-derived peptides that cross-react with human self-epitopes leading to tissue pathology. This phenomena is illustrated by rheumatic fever, a systemic autoimmune disease that follows infection with Streptococcus pyogenes. It is associated with high circulating titers of antistreptolysin (ASO) antibodies and positive bacterial cultures in the blood. Mimicry between group A streptococcus and host antigens in the heart and brain unfold in different ways ${ }^{[11]}$. Pathogenic circulating antibodies elaborated by human hosts against the bacterium cross-react with epitopes of the group A carbohydrate, $\mathrm{N}$ acetyl-beta-D-glucosamine present in heart valve 
endothelium is followed by a second wave of a T-cell mediated attack $^{[12]}$.

Two post-infectious streptococcal syndromes, Sydenham chorea (SC) and pediatric autoimmune neuropsychiatric disease associated with group A streptococcus (PANDAS) manifest abnormal movements and neuropsychiatric behaviors. In particular, SC occurs during rheumatic fever and is characterized by involuntary movements preceded by neuropsychiatric symptoms and emotional lability while PANDAS cases manifest tics and obsessive-compulsive disorder (OCD) ${ }^{[13]}$. Although there is a close relation between neuronal directed $\operatorname{IgG}$ antibodies in the serum and cerebrospinal fluid that target subcortical grey brain matter in cases of SC, the same cannot be said for PANDAS. However studies suggest that the dopamine D1 and D2 10 receptors may be important targets in both diseases [14] suggesting a homology of the two disorders.

Children with comprised immune systems, like those who are highly allergic, and many with chronic recurring sinus and throat infections, and enlarged tonsils are most at risk for harboring strep and developing PANDAS. Such children may be monitored by pediatricians even as they become neurologically symptomatic with tics and OCD disturbing family and school dynamics. Undiagnosed and untreated, such children may be referred to a mental health care provide to exclude a variety of disorders including attention deficit; disruptive, impulse control, and conduct disorders, autism, and even major depression and psychotic disorders until the diagnosis is correctly ascertained. The often finding of low baseline Ig levels in untreated cases suggests a state of acquired immune deficiency as a result of the postinfectious insult. In children with intravenous Ig therapy for persistent symptoms of PANDAS after intensive therapy with antibiotics, a low serum baseline levels of IgG ( $p<$ $0.0001)$ or IgG subclasses ( $\mathrm{p}<0.0003$ ) were associated with $100 \%$ remission of symptoms of PANDAS at 12 months $^{[15]}$. A multimodality therapeutic approach to PANDAS ${ }^{[16]}$ should also include anti-obsessional medication, cognitivebehavior therapy and prophylactic antibiotics as described at the PANDAS Physicians Network (PPN) (www.pandasppn.org).

By treating the underlying mechanism of post-infectious autoimmunity instead of the symptoms alone, PANDAS like other I-Cube disorders can be effectively managed and cured as illustrated by the case of Carlos.

Meet Carlos ${ }^{[1]}$, who exhibited behavioral changes in 2012 at age 7 when he struck his mother several times and spit at his father. Like most children his age he had recurrent strep throats and was treated with antibiotics. However while his throat improved, his behaviors 11 worsened and became violent. His parents consulted a child psychiatrist who treated him with fluoxetine (Prozac $\AA$ ) but that was complicated by emotional side effects. A neurologist later placed him on methylphenidate (Concerta $\left.{ }^{\circledR}\right)$, a popular medication used to treat attention deficit disorder which he continued intermittently until he seemed to improve. He had no further strep throats or symptoms of PANDAS until the end of summer camp in 2017, when seemingly out of nowhere, Carlos became unmanageable with vocal sniffing tics, obsessive compulsive and oppositional behaviors. Confused and frustrated, Carlos' parents brought him to see me. I ordered a PET MRI of the brain that showed hypometabolism of the hippocampi of both medial temporal lobes. This localized the area of the brain which is implicated in other autoimmune neuropsychiatric mood disorders ${ }^{[17]}$, is also the likeliest source of PANDAS neuropsychiatric manifestations. He was treated with intensive and prophylactic antibiotics and intravenous $\mathrm{Ig}$ therapy with improvement and has since been the sweet boy his parents recognize.

\section{Conflicts of Interest}

The author receives royalties for the sale of his books.

\section{Funding Statement}

There was no funding.

\section{References}

[1] Younger, David S. The Autoimmune Brain. A Five-Step Plan for Treating Chronic Pain, Depression, Anxiety, Fatigue, and Attention Disorders. New York: Rowman and Littlefield, 2019.

[2] Rappaport SM, Smith MT. Epidemiology. Environment and disease risks. Science, 330 (2010), pp. 460-461.

[3] Fousteri G, Dave Jhatakia A. Viral Infections and Autoimmune Disease: Roles of LCMV in Delineating Mechanisms of Immune Tolerance. Viruses. 2019; 11(10):885. Published 2019 Sep 21.

[4] Dunne A. Inflammasome activation: from inflammatory disease in infection. Biochem Soc Trans 2011; 39:669-673.

[5] Lupfer CR, Rodriques A, Kanneganti TD. Inflammasome activation by nucleic acids and nucleosomes in sterile inflammation.or is it sterile? FEBS J 2017; 284:2363-2374.

[6] Bogdanos DP, Smyk DS, Rigopoulou E, et al. Infectomics and autoinfectomics: a tool to study infectious-induced autoimmunity. Lupus. 2015; 24(4-5):364-373.

[7] Jeppesen R, Benros ME. Autoimmune Diseases and Psychotic Disorders. Front Psychiatry 2019; 10:131.

[8] MacQueen GM, Campbell S, McEwen BS, et al. Course of illness, hippocampal function, and hippocampal volume in major depression. Proc Natl Acad Sci USA 2003; 100:1387-1392. 
[9] Younger DS. Serial Brain Positron Emission Tomography Fused to Magnetic Resonance Imaging in Post-Infectious and AutoantibodyAssociated Autoimmune Encephalitis. David S. Younger, World Journal of Neuroscience, 2019, 9, 153-156.

[10] Lower R, Lower J, Kurth R. The viruses in all of us: characteristics and biological significance of human endogenous retrovirus sequences. Proc Natl Acad Sci USA 1996; 93:5177-5184.

[11] Younger DS, Bouboulis DA. Immune pathogenesis pediatric autoimmune neuropsychiatric disorders associated with group A ${ }^{\beta}$-hemolytic streptococcal infections (PANDAS). Int J Neurol Res 2015; 1:57.

[12] Ellis NMJ, Li Y, Hildebrand W, et al. T cell mimicry and epitope specificity of crossreactive $\mathrm{T}$ cell clones from rheumatic heart disease. J Immunol 2005; 175:5448-5456.

[13] Swedo SE, Leonard HL, Schapiro MB, et al. Sydenham's chorea: physical and psychological symptoms of St. Vitus dance. Pediatrics. 1993; 91:706-713.

[14] Cunningham MW. Streptococcus and rheumatic fever. Curr Opin Rheumatol 2012; 24:408-416.

[15] Younger DS, Mast PA, Bouboulis DA. Baseline immunoglobulin levels predict achievement of remission at one year following IVIg therapy. J Neurol Neurosurgy 2016; 3(2):122.

[16] Younger DS, Chen X. IVIg therapy in PANDAS: Analysis of the current literature. J Neurol Neurosurg 2016; 3(3):125.

[17] Younger DS. Autoimmune Encephalitides. World Journal of Neuroscience 2017; 7:327-361. 